\title{
Swelling equilibrium of dentin adhesive polymers formed on the water-adhesive phase boundary: Experiments and micromechanical model
}

\author{
Anil Misra ${ }^{1,2,3}$, Ranganathan Parthasarathy ${ }^{1,4}$, Qiang $\mathbf{Y e}^{1}$, Viraj Singh ${ }^{1,3}$, and Paulette \\ Spencer ${ }^{1,3}$ \\ ${ }^{1}$ Bioengineering Research Center, University of Kansas, Lawrence, KS, USA \\ ${ }^{2}$ Civil, Environmental and Architectural Engineering Department, University of Kansas, Lawrence, \\ KS, USA \\ ${ }^{3}$ Department of Mechanical Engineering, University of Kansas, Lawrence, KS, USA \\ ${ }^{4}$ Bioengineering Graduate Program, University of Kansas, Lawrence, KS, USA
}

\section{Abstract}

During their application to the wet, oral environment, dentin adhesives can experience phase separation and composition change which can compromise the quality of the hybrid layer formed at the dentin-adhesive interface. The chemical composition of polymer phases formed in the hybrid layer can be represented using a ternary water-adhesive phase diagram. In this paper, these polymer phases have been characterized using a suite of mechanical tests and swelling experiments. The experimental results were evaluated using granular micromechanics based model that incorporates poro-mechanical effects and polymer-solvent thermodynamics. The variation of the model parameters and model-predicted polymer properties has been studied as a function of composition along the phase boundary. The resulting structure-property correlations provide insight into interactions occurring at the molecular level in the saturated polymer system. These correlations can be used for modeling the mechanical behavior of hybrid layer, and are expected to aid in the design and improvement of water-compatible dentin adhesive polymers.

\section{Keywords}

dentin adhesive; phase diagram; absorption; swelling; micromechanics; crosslink density; elastic modulus

\section{Introduction}

Polymers formed from hydrophilic/hydrophobic monomer pairs have been used in a variety of biomedical applications such as bioadhesives, artificial organs, prostheses, drug delivery systems and orthopedic materials [1-11]. The polymer properties are strongly correlated to its chemical composition and network structure [12-14]. Particularly, the phase separation,

\footnotetext{
C 2013 Acta Materialia Inc. Published by Elsevier Ltd. All rights reserved.

Corresponding Author: Dr. Anil Misra, Professor, Civil, Environmental and Architectural Engineering Department, The University of Kansas, Learned Hall, 1530 W. 15th Street, Lawrence, KS 66045-7609, Ph: (785) 864-1750, Fax: (785) 864-5631, amisra@ ku.edu.

Publisher's Disclaimer: This is a PDF file of an unedited manuscript that has been accepted for publication. As a service to our customers we are providing this early version of the manuscript. The manuscript will undergo copyediting, typesetting, and review of the resulting proof before it is published in its final citable form. Please note that during the production process errors may be discovered which could affect the content, and all legal disclaimers that apply to the journal pertain.
} 
water sorption, associated swelling, water diffusion, mechanical properties and enzymatic resistance of these materials significantly influence their functional performance. The dentin adhesive systems used in composite tooth restorations, like many other bioadhesive systems, are also composed of hydrophobic/hydrophilic organic monomer mixtures. In composite tooth restorations, adhesive systems formed of hydrophilic/hydrophobic monomer mixtures infiltrate into the wet, demineralized collagen of the tooth to form a collagen-adhesive composite known as the hybrid layer $[15,16]$. One of the major factors that affects the stability and durability of the hybrid layer is inadequate penetration of the adhesive and its physical separation into individual phases $[17,18]$. The presence of water in wet demineralized dentin is a major detrimental factor for dentin adhesives used to bond composite restorations. It is well accepted that the hybrid layer is the weakest link in the adhesive-dentin bond [19].

In dentin adhesive systems the hydrophobic/hydrophilic monomer pair serves the following functions: a) in the monomer state, the adhesive hydrophilicity aids in its integration with the wet, demineralized dentin matrix, while b) in the polymerized state, adhesive hydrophobicity leads to lower sorption of oral fluids and endows adequate stiffness to minimize swelling [20-24]. Thus, to provide durable function in the mouth, the optimal dentin adhesive must possess a hydrophilic/hydrophobic balance. However, in the wet, oral environment, dentin adhesives can experience composition change and phase separation. The penetration of the adhesive into the wet demineralized dentin may take place by a combination of diffusion and convection resulting from external energy during adhesive application on the dentin substrate [25-27]. Since the hydrophobic component is also more viscous, it transports slower through both diffusion as well as convection, as compared to the hydrophilic component. Hence, subsequent photo-polymerization results in an adhesivecollagen hybrid layer where the composition of the adhesive changes spatially, particularly along the depth of the hybrid layer. Both, phase separation [28, 29] and limited infiltration of the hydrophobic component along the depth of the hybrid layer [17, 18], have been experimentally observed in dentin adhesive interfaces.

The possible monomer phases formed in the hybrid layer by the mixing of the hydrophobic/ hydrophilic adhesive monomer pair with water can be represented by the phase boundary of an adhesive-dentin ternary phase diagram [28]. Phase separation and adhesive composition changes in the monomer state affect both the hydrophilicity and stiffness of the adhesive polymer phases. The hydrophilicity and stiffness are the major factors that control the chemo-mechanical behavior of the adhesive polymer [30-37], which has a profound influence on the load transfer mechanism at the dentin-adhesive $(\mathrm{a} / \mathrm{d})$ interface and its fatigue life [38-41]. Further, it has been observed that the visco-plastic properties of the adhesive change anomalously with water content under loading [42]. In addition, the hydrophilicity and cross-link density have been identified to be two important independent variables which affect the water sorption and diffusion into methacrylate-based cross-linked polymers [43]. These adhesive polymer phases are not conventional hydrogels, in that they do not behave as rubbery elastomers at room temperature, except at very low crosslinker concentrations. Starting from a dry condition, when they absorb water and swell, a part of the interchain interactions dissociate, making them partly rubbery. However, conventional constitutive models are either designed for rubbery elastomers [44-47], or for glassy solids [48]. The mixed nature of our polymer phases necessitates a model which can account for (a) the energetic contribution to stiffness (b) the interaction between polymer and water leading to sorption and (c) the poromechanical effects arising from fluid pressure.

The aim of this paper is to characterize and understand the variation of polymer phase chemo-mechanical properties along the adhesive-dentin phase boundary using an appropriate constitutive model. A suite of mechanical tests and swelling experiments were 
performed on five different polymer formulations whose water-hydrophilic-hydrophobic compositions lie close to the phase boundary. A micro-chemo-poro-mechanical model was developed to describe the swelling behavior of these polymer formulations. The experimental data was used to obtain the variation of model parameters with chemical composition. Additional experimental evidence was collected to independently confirm the variation in the model parameters. The model parameters were then used to predict estimates of Poisson's ratio and bulk modulus. The model parameters and predicted results were qualitatively and quantitatively studied with respect to the chemical composition along the phase boundary. The resulting structure-property relationships give insight into the influence of chemical composition on the variation of chemo-mechanical properties along the phase boundary.

\section{Materials and experimental methods}

\subsection{Materials}

Methacrylate based dentin adhesive monomer formulations were prepared as mixtures of BisGMA (Bisphenol A glycerolate (1 glycerol/phenol) dimethacrylate), 2hydroxyethylmethacrylate (HEMA, 99\%) (Sigma Chemical Co., St. Louis, MO, USA.) and deionized water. The chemical structures of the co-monomers are shown in Table 1. The following three-component visible light photoinitiator (all from Aldrich, Milwaukee, WI) was used in this work: camphoroquinone (CQ, $0.5 \mathrm{wt} \%)$, ethyl-4-(dimethylamino)benzoate (EDMAB, $0.5 \mathrm{wt} \%$ ) and diphenyliodonium hexafluorophosphate (DPIHP, $0.5 \mathrm{wt} \%$ ). The concentration of the photoinitiator components was calculated with respect to the total amount of monomer. All materials were used as received.

\subsection{Preparation of neat adhesive resins}

Monomer mixtures in the following mass ratios of HEMA to BisGMA were added along with a 3-component photo-initiator system ( 0.5 mass percent of camphorquinone (CQ), ethyl-4-dimethylamino benzoate (EDMAB) and diphenyl iodonium phosphate (DPIHP)) into brown vials: a) $92.5: 7.5$ b) 80:20 c) 75:25 d) 70:30 e) 60:40 f) 45:55, g) 30:70 and h) 85:15 (HEMA:BisGMA). The monomer-initiator mixtures in the brown vials were prepared using vortex mixers and shakers to obtain a homogeneous adhesive resin. The neat resins are represented on the ternary phase diagram using squares as shown in Figure 1. The black arrow indicates the direction of increasing BisGMA content in the resin. A weighing balance with a resolution of $0.01 \mathrm{mg}$ (Mettler Toledo, X205 dual range) was used to weigh each component and was used for all mass measurements described henceforth.

\subsection{Determination of water miscibility of neat adhesive resins}

About $1 \mathrm{~g}$ of each neat resin was weighed into a brown vial and water was added in increments of approximately $0.01 \mathrm{~g}$ until the mixture was visually observed to be turbid. The percentage of water in the mixture was noted $\left(w_{l}\right)$. The mixture was then back-titrated using the neat resin till the turbidity disappeared and the percentage of water in the mixture was noted $\left(w_{2}\right)$. The water solubility was calculated as the average of $w_{1}$ and $w_{2}$. The procedure is represented on the ternary phase diagram as moving along the line of constant monomer ratio starting at the neat resin (square point) towards the intersection with the phase boundary line, which represents the limit of miscibility for that monomer ratio.

\subsection{Preparation of polymer samples from monomer-water formulations on phase boundary}

Water equivalent to about 2-3 percentages below the solubility limit is added to each neat resin and mixed till fully dissolved (see Table 2). The water content was chosen to lie within the solubility limit to minimize polymerization induced phase separation at the scale of this study. The composition of these formulations has been represented using circles on the 
ternary phase diagram as shown in Figure 1 . The samples in Figure 1 were prepared at $24{ }^{\circ} \mathrm{C}$ and ambient pressure. Square beams with a side of $1 \mathrm{~mm}$ and a length of at least $10 \mathrm{~mm}$ were prepared for each formulation by casting these prepared adhesive resins into glasstubing molds (Fiber Optic Center Inc, \#CV1012, Vitrocom Round Capillary Tubing of Borosilicate Glass). The resins were injected into the tubing using a micro-pipette and light polymerized with a LED light curing unit of effective irradiance $250 \mathrm{~mW} / \mathrm{cm}^{2}$ and area 6.25 $\mathrm{mm}^{2}$ for 40s (LED Curebox, Prototech, and Portland, OR). The effective irradiance was provided by the manufacturer based upon the intensity of the blue LED used in the box. The wavelength of light for the LED curebox is around $470 \mathrm{~nm}$. The polymerized samples were stored in dark at room temperature for two days to provide adequate time for post-cure polymerization. The samples were subsequently extracted from the glass tubing and stored in a vacuum oven in the presence of a drying agent (freshly dried silica gel) at $37^{\circ} \mathrm{C}$.

\subsection{Absorption and swelling experiment}

Multiple beam specimens were used to study the water sorption behavior of each formulation. Distilled, deionized water (HPLC grade, W5SK-4, Fisher Scientific, Fair Lawn, NJ, USA) was used throughout the experiments. The specimens were first pre-washed by immersing in water for 15 days to remove any leachable matter. During the pre-washing process, timed mass change of the samples was recorded. The time interval for data collection varied from 10 minutes during the beginning of the experiment to 24 hours. After each time interval, the specimens were retrieved, blotted dry to remove excess liquid, weighed in air, and returned to the water bath. At the end of 15 days, the beam specimens were placed into a vacuum chamber for drying until they reached constant mass. The specimens were then re-immersed in water and stored at room temperature. These specimens were used for the post-wash data collection. Again the time interval for data collection varied from 10 minutes during the beginning of the experiment to 24 hours. After each time interval, the specimens were retrieved, blotted dry to remove excess liquid, weighed in air, weighed in water, and returned to the water bath. The measurement under water was performed within about 10 seconds to avoid any absorption of water during the process. The mass under water is equal to the mass in air minus the buoyancy force exerted by the water. The volume of the sample was calculated using the mass in air and water as shown below.

$$
V=\alpha \frac{m_{a}-m_{w}}{\rho_{w}-\rho_{a}}
$$

where $V$ is the volume of the sample, $m_{a}$ is the mass of the sample in air, $m_{W}$ is the mass of the sample in water, and $\rho=0.99985$ is a factor to account for air bouyancy. $\rho_{W}=1 \mathrm{~g} / \mathrm{cm}^{3}$ and $\rho_{a}=0.0012 \mathrm{~g} / \mathrm{cm}^{3}$ are the densities of water and air respectively. The sample mass density $\rho$ was calculated as follows.

$$
\rho=\frac{m_{a}}{m_{a}-m_{w}}\left(\rho_{w}-\rho_{a}\right)+\rho_{a}
$$

The measured fractional mass change in air, $\Delta m_{a}$, and in water, $\Delta m_{w}$, was obtained as

$$
\left(\Delta m_{a}\right)_{\text {meas }}=\frac{m_{a}-m_{a 0}}{m_{a 0}} ; \quad \text { and } \quad\left(\Delta m_{w}\right)_{\text {meas }}=\frac{m_{w}-m_{w 0}}{m_{w 0}}
$$

and the volume change expressed as the Jacobian of the deformation, $J$ was calculated as follows: 


$$
J=\frac{V}{V_{0}}
$$

where $m_{a 0}, m_{\omega 0}$ and $V_{O}$ are the initial sample mass measured in air, initial sample mass measured in water and volume, respectively.

\subsection{Dynamic Mechanical Analysis}

The measurement of glass transition temperature and storage modulus in the rubbery state was carried out using dynamic mechanical analysis (DMA Q800, TA Instruments, New Castle, USA) in a 3-point bending configuration. The analysis has been described previously [49]. The frequency used to measure the storage modulus was $1 \mathrm{~Hz}$ with amplitude of $15 \mu \mathrm{m}$ and a preload of $0.01 \mathrm{~N}$. The applied displacements and measured loads were converted to stresses and strains using the equations of elastic beam theory for long slender beams undergoing small deformations. The storage modulus was measured across $0^{\circ} \mathrm{C}$ to $250^{\circ} \mathrm{C}$ using a temperature sweep. The temperature sweep was conducted at $3^{\circ} \mathrm{C} / \mathrm{min}$. The glass transition temperature was identified from the peak of the tan $\delta$-temperature curve. The storage modulus decreased with temperature and reached an asymptote at the glass transition temperature as shown in the supplementary Figures S1 and S2. This asymptotic storage modulus was taken to be the apparent rubbery modulus of the polymer. The covalent crosslink density was calculated based on Flory's rubber elasticity theory $[45,50]$ as shown below:

$$
\nu_{e}=\frac{E^{\prime}}{3 R T}
$$

where $E$ is the apparent rubbery modulus, $T$ is the temperature at which the rubbery plateau of the polymer begins, and the universal gas constant $R=8.31 \mathrm{~J} \mathrm{~K}^{-1} \mathrm{~mol}^{-1}$. Eq. (5) is based on the rubber elasticity theory and is not valid for high degrees of cross linking, but has been used when the storage modulus in the rubbery region is in the range of $2 \times 10^{6}$ and $2 \times 10^{8} \mathrm{~Pa}$ [50]. Eq. (5) has been used widely to estimate the crosslink density of polymeric materials. In addition, the stoichiometric crosslink density $v_{S}$ in moles $/ \mathrm{m}^{3}$ was calculated from the mole percentage of the cross-linker and the degree of conversion as follows, assuming that the degree of conversion to be uniform for both BisGMA and HEMA [51]

$$
\nu_{s}=\frac{100 b(D C) \rho}{M_{b}}
$$

where $b$ is the percentage of BisGMA in the polymer, DC is the percentage degree of conversion shown in Figure $\mathrm{S} 3, \rho$ is the polymer density and $M_{b}$ is the molar mass of BisGMA.

\subsection{Mechanical testing}

Mechanical tests were performed using a three-point bending apparatus with $10 \mathrm{~mm}$ beam span on a Bose Electroforce 3200 tester. Completely saturated and swollen samples that were subjected to absorption and swelling experiments (section 2.6) were used for this test. Loading was applied at a rate of $0.001 \mathrm{~mm} / \mathrm{sec}$, or equivalently in terms of the elastic strains at the rate of $75 \mu$ strain/sec. Average stress-strain curves $(n=3)$ were obtained and used to calculate the elastic modulus from the linear region ( $\leq 1 \%$ strain) using a straight line fit for each formulation under dry and wet conditions, respectively. The goodness of fits ranged from $92.9 \%$ for formulation with low BisGMA content to $99.9 \%$ for medium to high BisGMA content. 


\section{Mathematical Model}

A number of theories to model the elasticity of a polymer network have been developed, such as the kinetic or statistical theory of rubber elasticity, and the phenomenological Mooney-Rivlin type models and their variations [47]. It is well accepted that the macroscale properties of a material are significantly influenced by the interaction between representative units. Constitutive models that relate the externally applied action to the parameters that govern the interaction between representative units have an advantage for describing the mechanical behavior of polymer networks because they enable mechanical responses to be related to the composition (see for example discussion in [47]). In many polymeric systems, the relevant representative unit can be modeled as grains or blobs. In this case, polymers can be described as assemblies of grains or blobs interacting through entanglements or crosslinks; where each blob retains the Gaussian characteristics of a Flory chain at low crosslink densities and non-Gaussian characteristics at higher crosslink densities as depicted by De Gennes [52]. Results of small-angle neutron scattering have shown that polymer gels have similar characteristics as dense fluids of hard spheres [53]. Recent simulations agree with this granular or blob description of polymers [54]. Similar granular descriptions have also been associated with acoustic dispersion phenomena, wherein the grain size in materials such as polymers has been interpreted as the free path between acoustic scattering sites [55]. In the granular descriptions, the grains are viewed as atom aggregations in which the intra-granular atomic interactions have a qualitatively different nature than the inter-granular interactions. For example in cross-linked polymers, the intra-granular interactions are the covalent bonds within the polymer chains. The intergranular interactions, on the other hand, may comprise of covalent crosslinks and the noncovalent hydrogen-bonds, van der Waals interactions and physical entanglements. In these cases, the overall inter-granular interaction may be viewed in a statistical sense. In principle, the inter-granular interaction may be treated from atomistic viewpoint; however such an approach can be computationally intractable for cross-linked polymeric systems with billions of atoms and ill-defined structure. From a practical viewpoint, we can formulate appropriate functions to describe the inter-granular interactions in which the essential subgranular scale mechanisms are represented and the interaction function parameters can be obtained from the experimental data. Therefore, in this paper, we treat the adhesive polymer to possess a pseudo-granular structure in which the grain-interactions represent the average behavior of the covalent bonds within the chain, the covalent cross-links between segments in two different polymer chains, non-covalent hydrogen bonds, van der Waals interactions, entanglements, and other physical interactions. Granular micromechanics theory has been used to derive bounds of elastic moduli for granular materials using inter-particle contacts $[56,57]$ and more recently, to derive constitutive equations for rate-dependent visco-damage behavior, including loading induced anisotropy, based upon inter-granular forcedisplacement relationships [58]. Similar approaches have been used for modeling articular cartilage which can be described as a fiber reinforced polymeric gel $[59,60]$.

The proposed approach provides a feasible meso-scale method to relate the macro-scale properties to molecular-scales for materials whose micro-structure and local interactions are ill-defined [58,61]. The term meso-scale has been used to refer to a scale smaller than the micro-scale but much larger than the size of a polymer segment (molecular scale), such that a representative volume element at the meso-scale contains several thousand polymer segments. We modeled the wet adhesive polymer using a three-step procedure by applying a) granular micromechanics [56] to model the elastic behavior of the polymer network, b) a self-consistent homogenization of the (homogenized) polymer network with the pore space to obtain the homogenized porous polymer stiffness, and c) the poro-mechanical approach to obtain the behavior of the saturated polymer by superposing the (homogenized) porous polymer with the interstitial water. A schematic depiction of the three-step modeling 
procedure is shown in Figure 2(a) through 2(c). In addition, a schematic depiction of the idealization for cases of low and high cross-link density is shown in Figure 3(a) and (b), respectively. The BisGMA monomer is expected to act as a crosslink when the two double bonds of the BisGMA molecule form active centers for polymerization, while HEMA chains between the crosslinks have a high freedom of movement in the interstitial water. At low crosslink densities, the crosslinks are spaced far apart and the influence of non-covalent interactions is weaker. On the other hand, for high crosslink densities, the chain lengths are much shorter and the non-covalent interactions are stronger. The average inter-granular stiffness produced by these interactions is modeled using the micro-mechanical parameters elaborated in the next section.

\subsection{Micro-poro-mechanical model}

In the first step (see Figure 2(a)), the theoretically dense wet polymer network was homogenized using the granular micromechanics approach which assumes the system to be composed of grains interacting in both the normal and shear directions. In this approach, the stress tensor was obtained in terms of the tensor product of the interaction force vector and the vector joining the centroid of pseudo-grains [58]. The resultant expression for the stress tensor has similarities to the microscopic stress description utilized for reptation models of polymers (see for example Section 5.3 [62]). The Kramers-Kirkwood relation and the derived microscopic stress based on the Rouse model is also similar in form to the stress tensor derived from a granular micromechanics approach [56, 63-66].

Using the granular micromechanics approach, the following expression for Young's modulus and Poisson's ratio can be derived for isotropic systems subjected to isotropic loading, under the so called "static hypothesis" for expressing inter-granular forces in terms of the stress tensor [56].

$$
\begin{gathered}
E^{s}=10 A^{s}\left(\frac{\alpha^{s}}{2+3 \alpha^{s}}\right) \\
\nu^{s}=\frac{1-\alpha^{s}}{2+3 \alpha^{s}}
\end{gathered}
$$

where $E^{s}$ and $v^{s}$ are the elastic modulus and Poisson's ratio of the polymer network, $A^{S}$ is a meso-scale parameter proportional to the average inter-granular stiffness in the normal direction and the number density of inter-granular interactions, and $a^{S}$ represents the ratio of the average transverse to the average normal inter-granular stiffness. For instance, in a flexible linear polymer with no non-covalent interactions between chains, $\alpha^{s}$ is close to 0 leading to a Poisson's ratio $v^{\varsigma}$, close to 0.5 , as observed for hydrogels and rubbers [67-70]. A completely crosslinked structure such as diamond where the inter-atomic transverse and normal stiffness are nearly equal has a Poisson's ratio close to zero [71]. Formulae relating other forms of macro-scale material properties to meso-scale parameters are given in Table 3. The "static hypothesis" approach is derived by relating the macro-scale strain to the local displacements through least square fitting [56] in order to capture the non-affine local deformations observed in these material systems [72].

In the second step (see Figure 2(b)), the polymer network was homogenized with the porous space using the self-consistent approximation [73] to obtain the $4^{\text {th }}$-order stiffness tensor $C_{i j k l}$ of the porous polymer as shown below. 


$$
C_{i j k l}=C_{i j k l}^{s}-\varphi C_{i j m n}^{s} H_{m n k l}^{\varphi}
$$

where $H^{\varphi}$ is the strain concentration tensor in the pore space determined by using the equivalent inclusion technique [74], and $\varphi$ is the porosity of the polymer. Similar methods have been used in more general settings for nonlinear problems $[75,76]$. The stiffness tensor $C_{i j k l}^{s}$ was written in terms of $E^{S}$ and $v^{S}$ given in Eqs. (7) and (8) as

$$
C_{i j k l}^{s}=\lambda^{s} \delta_{i j} \delta_{k l}+\mu^{s}\left(\delta_{i k} \delta_{j l}+\delta_{i l} \delta_{j k}\right)
$$

where $\lambda^{\mathrm{s}}$ and $\mu^{s}$ can be determined from formulae in Table 3, and $\delta_{k l}$ is the Kronecker delta. We have used the tensor notation for the development of the mathematical model, thus the subscripts in this section follow the tensor summation convention. For the saturated swollen polymer, the pore spaces were assumed to be spherical for simplicity in the absence of welldefined morphological information. The Eshelby tensor $S$ for spherical inclusion in isotropic medium is given as [77]

$$
S_{i j k l}=\frac{(5 \nu-1)}{15(1-\nu)} \delta_{i j} \delta_{k l}+\frac{(4-5 \nu)}{15(1-\nu)}\left(\delta_{i k} \delta_{j l}+\delta_{i l} \delta_{j k}\right)
$$

where $v$ is the Poisson's ratio of the homogenized porous polymer. The strain concentration tensor $H^{\varphi}$ for the pore space is then calculated as follows [73]:

$$
H_{i j k l}^{\varphi}=\left(I_{i j k l}-S_{i j k l}\right)^{-1}
$$

where $I=\left(\delta_{i j} \delta_{k l}\right)$ is the identity tensor.

In step three (see Figure 2(c)); we superposed the homogenized porous polymer phase with the fluid phase following the procedure described in [73] after considering large deformation. We thus obtained the incremental overall constitutive equation for the saturated polymer in a poroelastic framework as follows.

$$
\begin{gathered}
\dot{\sigma}_{i j}=-\delta_{r s} L_{r s} \bar{\sigma}_{i j}+C_{i j k l} \dot{\varepsilon}_{k l}+\bar{\sigma}_{j m} L_{i m}+\bar{\sigma}_{i m} L_{j m}-B_{i j} \dot{p} \\
\dot{\zeta}=B_{k l} \dot{\varepsilon}_{k l}+\Gamma \dot{p}
\end{gathered}
$$

where $\sigma_{i j}$ is the stress rate, $\sigma_{i j}^{-}$is the stress in the polymer phase, $L_{i m}$ is the velocity gradient of the saturated polymer, $\epsilon_{k l}$ is the strain rate, $\dot{p}$ is the fluid pressure rate, $\zeta^{k}$ s the volumetric rate of water uptake with respect to the volume of the saturated polymer, and $B_{i j}$ and $\Gamma$ are the poromechanics constants. $B_{i j}$ and $\Gamma$ were obtained in a straightforward manner using self-consistent homogenization [61] as:

$$
\begin{gathered}
B_{i j}=\varphi \delta_{m n} H_{m n i j}^{\varphi} \\
\Gamma=B_{k l}\left(C_{i j k l}^{s}\right)^{-1}\left(B_{k l}-\varphi \delta_{k l}\right)+\frac{\varphi}{K_{f}}
\end{gathered}
$$

where $K_{f}$ is the bulk modulus of water. The poromechanics constant $B$ measures the ratio of water volume squeezed out to the volume change of the saturated polymer under (idealized) 
conditions of flow under zero pressure. $B_{i j}$ is called the effective stress coefficient and represents the proportion of the applied load carried by the fluid. $\Gamma$ is a compliance parameter which measures the amount of water which can be forced into the saturated polymer under pressure while the volume is kept constant. $\Gamma$ is a measure of the combined fluid-solid compressibility. It is noteworthy that the value of $B_{i j}$ is not unity and both $B_{i j}$ and $\Gamma$ depend on the stiffness of the porous polymer and the polymer network. Under the assumption of negligible change in water density, $\zeta^{Z}=\gamma_{W}^{A}$, where $\gamma_{W}^{A}$ is the increment in water volume fraction in the saturated polymer. The approach outlined above can be used to develop models for time-dependent deformations [78] or for including higher gradient effects due to capillarity and thin boundary layers that occur in these porous materials [7981].

\subsection{Chemical potential of the saturated polymer}

Water absorption and swelling is determined by the mechanical properties as well as the chemical potential of the water in the polymer. The chemical potential of water in a polymer relative to chemical potential of pure water has been derived by [45]. Here, we used a modified form written as:

$$
\mu=R T\left[\ln \left(\gamma_{w}\right)+\left(1-\gamma_{w}\right)+\chi\left(1-\gamma_{w}\right)^{2}\right]+\Psi\left(\gamma_{w}-V_{\varphi 0}\right) \bar{p} \frac{M_{w}}{\rho_{w}}
$$

where $\mu$ is the chemical potential difference between the water inside the polymer and pure water, henceforth referred to as chemical potential, $R$ is the universal gas constant $(=8.314$ $J K^{-1} \mathrm{~mol}^{-1}$ ), $T$ is the temperature, $\gamma_{W}$ is the water volume fraction, $p$ is the effective fluid pressure in the polymer given as $p=\int B_{i j} \delta_{i j} p, \frac{M_{w}}{\rho_{w}}$ is the partial molar volume of water in the polymer, $M_{W}$ the molecular weight and $\rho_{W}$ the mass density of water, $X$ is the polymerwater interaction parameter, $V_{\varphi 0}$ is the initial free volume in the polymer and $\Psi_{\text {is }}$ the Heaviside step function such that $\Psi\left(\gamma_{W}-V_{\varphi 0}\right)=0$ when $\gamma_{W}<V_{\varphi 0}$ and $\Psi\left(\gamma_{W}-V_{\varphi 0}\right)=1$ when $\gamma_{W} \geq V_{\varphi 0}$. The effective fluid pressure $p$ a t equilibrium when the chemical potential difference vanishes, $\mu=0$, is identified as the osmotic pressure. We note that the first term in Eq. (17) represents the free energy resulting from the entropy and enthalpy of mixing, while the second term represents the contribution from elastic deformation. The Heaviside step function was introduced in the second term to account for the initial free volume, $V_{\varphi 0}$, defined as that fractional volume of the polymer which needs to be saturated before the interstitial water develops pressure as the result of the elastic swelling of the polymer structure. In literature, the free volume has been defined as the volume not occupied by the polymer molecules but constituting a part of the bulk volume of the overall polymer solid or polymer/diluent system [82]. The free volume in a polymer has also been defined equivalently as the volume occupied by openings between the polymer chains where the solvent molecules can pass through [82, 83]. Our definition is consistent with those from literature since the fluid pressure and corresponding stress in the polymer is created only when there is resistance to any further increase in space between the chains beyond the initially empty space.

\subsection{Swelling and elastic modulus of saturated polymer}

We assume that the swelling under stress-free condition, wherein the increment of external stress, $\sigma_{i j}^{7}=0$, is isotropic, that is $\epsilon_{i j}^{D}=\epsilon \delta_{i j}$ and $B_{i j}=B \delta_{i j}$. Furthermore, for the swelling experiments performed on beams whose length is much larger than the cross sectional dimensions plane strain conditions may be assumed. Solving Eq. (13) and (14) under these conditions, we obtained the following relationship applicable to our experiments 


$$
\begin{aligned}
& \dot{p}=\left[\frac{C_{1111}+C_{1122}}{2 B^{2}+\Gamma\left(C_{1111}+C_{1122}\right)}\right] \dot{\gamma}_{w} \\
& \dot{\varepsilon}=\left[\frac{B}{2 B^{2}+\Gamma\left(C_{1111}+C_{1122}\right)}\right] \dot{\gamma}_{w}
\end{aligned}
$$

where the stress correction terms involving the velocity gradient, $L_{i j}$, vanish for the condition of plane strain. The volume change expressed as the Jacobian of the deformation, $J$ is related to the isotropic strain as

$$
\frac{\dot{J}}{J}=2 \dot{\varepsilon} \quad \text { (20) }
$$

Further, we note that the rate of loading for mechanical testing performed in this study is orders of magnitude faster than the rate of water diffusion (or drainage) in the beam samples. Therefore, the loading can be considered as undrained and $\zeta=0$. Solving Eq. (13) and (14) for this condition gives a relationship for the undrained stiffness in term of the drained stiffness $C_{i j k k}$, used in Eqs. (18) and (19), and poromechanics constants $B_{i j}$ as follows

$$
C_{i j k l}^{u}=C_{i j k l}+\frac{B_{i j} B_{k l}}{\Gamma}
$$

\subsection{Numerical model calibration}

Eqs. (17) through (21) allows us to relate the macroscopic experimental measurements, that is the undrained elastic modulus, the mass change in air, the mass change in water and the volume change to the local (meso-scale) parameters $A^{S}, V_{\varphi 0}$ and $\chi$. However, a numerical solution of these coupled nonlinear equations is required for the calibration of the mesoscale parameters. To this end, we minimized the following objective error function

$e\left(A^{s}, V_{\varphi 0}, \chi\right)=\left(\frac{\Delta m_{a}-\left(\Delta m_{a}\right)_{\text {meas }}}{\left(\Delta m_{a}\right)_{\text {meas }}}\right)^{2}+\left(\frac{\Delta m_{w}-\left(\Delta m_{w}\right)_{\text {meas }}}{\left(\Delta m_{w}\right)_{\text {meas }}}\right)^{2}+\left(\frac{J-J_{\text {meas }}}{J_{\text {meas }}}\right)^{2}+\left(\frac{E^{u}-E_{\text {meas }}^{u}}{E_{\text {meas }}^{u}}\right)^{2}$

subject to the constraints

$$
A^{s}<0,0<V_{\varphi 0}<1, \chi>0
$$

where the change of mass in air

$$
\Delta m_{a}=\frac{\rho_{w} V_{\varphi}}{m_{a 0}},
$$

the change of mass in water 


$$
\Delta m_{w}=\frac{\rho_{w}\left(V_{\varphi}-V+V_{0}\right)}{m_{w 0}}
$$

the jacobian $\mathbf{J}$ is given by Eq. (20), and the undrained elastic modulus is given by Eqs. (15), (16) and (21). We note that the parameter $a^{s}$ is not included in the error function to avoid multiple solutions since only a single mechanical property measurement is available. Additional mechanical property measurements will be needed for unique determination of parameter $a^{S}$.

Under saturation the water volume fraction, $\gamma_{w}=\varphi=\frac{V_{\varphi}}{V}$, and at equilibrium the chemical potential given by Eq. (17) must vanish, thus yielding the required relationship for determining $V, V_{\varphi} J$ and $E^{u}$, that are needed in Eq. (22). However, the effective fluid pressure, $p$, the drained stiffness, $C_{i j k l}$, and the poromechanics constants $B_{i j}$ and $\Gamma$ are unknown at equilibrium. Further, the relationship between the effective fluid pressure and the water volume fraction given by Eq. (18) is in an incremental form. Therefore, Eq. (17) was solved incrementally for the equilibrium condition, $\mu=0$. At each increment, the water volume fraction and effective pressure in Eq. (17) were solved using the iterative Newton's method for which we defined the residual at the end of increment $t+\Delta t$ as follows

$$
f\left(\gamma_{w}^{t+\Delta t}\right)=\mu^{t+\Delta t}-\left(R T\left[\ln \left(\gamma_{w}^{t+\Delta t}\right)+\left(1-\gamma_{w}^{t+\Delta t}\right)+\chi\left(1-\gamma_{w}^{t+\Delta t}\right)^{2}\right]+\bar{p}^{t+\Delta t} \frac{M_{w}}{\rho_{w}}\right)
$$

and expanded in Taylor's series as:

$$
f\left(\gamma_{w}^{t+\Delta t}\right)=f\left(\gamma_{w}^{t+\Delta t}\right)^{i}+\Delta \gamma_{w}^{t+\Delta t}\left(\frac{\partial f}{\partial \gamma_{w}^{t+\Delta t}}\right)^{i}
$$

where $\Delta \gamma_{w}^{t+\Delta t}$ is the correction in the $i$-th iteration of increment $t+\Delta t$. Setting the residual in Eq. (27) to zero, we get

$$
\Delta \gamma_{w}^{t+\Delta t}=\frac{f\left(\gamma_{w}^{t+\Delta t}\right)^{i}}{K_{T}^{i}}
$$

where the tangent

$$
K_{T}^{i}=-\left(R T\left[\frac{1}{\gamma_{w}^{t+\Delta t}}-1-2 \chi\left(1-\gamma_{w}^{t+\Delta t}\right)\right]+\Omega \frac{M_{w}}{\rho_{w}}\right)
$$

$\Omega=B\left[\frac{C_{1111}+C_{1122}}{2 B^{2}+\Gamma\left(C_{1111}+C_{1122}\right)}\right]$, and the increment of effective fluid pressure $\Delta p^{t 7 \Delta t}$ was calculated as $\Delta \bar{p}^{t+\Delta t}=\Omega \Delta \gamma_{w}^{t+\Delta t}$. At the end of the $i$-th iteration, we updated the water volume fraction as follows till convergence

$$
\gamma_{w}^{t+\Delta t}=\gamma_{w}^{t+\Delta t}+\Delta \gamma_{w}^{t+\Delta t}
$$

The above numerical scheme was implemented using the following 3 steps: 
Step 1 To initiate the calculations, we utilized the following initial estimates of meso-scale parameters: (1) $A^{s}=k E^{u}$, where $E^{u}$ is the undrained modulus obtained from the mechanical experiment, and $k$ is obtained from the relationship between $E$ and $A$ in Table 3 for assumed value of $a^{s}\left(k=5\right.$ for $a^{s}$ $=1.0$ and $k=13$ for $\alpha^{s}=0.2$ ); (2) the initial free volume, $V_{\varphi 0}$, is taken as a small number typically $<0.1$; and (3) $X=0.5$ which are similar to the published results for polyHEMA $[84,85]$.

Step 2 For the case of $\gamma_{W}<V_{\varphi 0}$, that is prior to the saturation of the initial free volume, the water pressure is zero and chemical potential, $\mu$, can be obtained from Eq. (17) directly for given $\gamma_{W}$. For $\gamma_{W}=V_{\varphi 0}$ the chemical potential $\mu^{\rho}$ is also obtained from Eq. (17) by substituting $p^{0-}=0$. For $\gamma_{W} \geq V_{\varphi \varphi}$, the solution was based upon the procedure outlined in Eqs. (26)-(30), where the chemical potential was updated using a uniform increment, $\Delta \mu=-\frac{\mu^{0}}{N}$, and $\mathrm{N}$ was chosen to be sufficiently large to obtain converged solutions with minimal iterations. For each increment and within each iteration, the values of $C_{i j k}$, $B_{i j}$, $\Gamma$ were updated using Eq. (9), Eq. (15) and Eq. (16) where $\varphi=\gamma_{w}$. We note that Eq. (9) is non-linear in $\varphi$ since $H_{i j k l}^{\varphi}$ and $S_{i j k l}$ depend on the Poisson's ratio, $v$, therefore, Eqs. (9), (11) and (12) were simultaneously solved using an optimization scheme, such as Levenberg-Marquardt scheme to determine, $v$, and subsequently $C_{i j k}, B_{i j}$ and $\Gamma$.

Step 3 Once the equilibrium values of $\gamma_{w}, p,{ }^{-} C_{i j k k}, B_{i j}$, $\Gamma$ were obtained at $\mu^{t+\Delta t}=0$, the change of mass in air (Eq. (24)), change of mass in water (Eq. (25)), Jacobian of deformation and undrained elastic moduli were calculated at equilibrium and substituted into Eq. (22) to calculate the error $e\left(A^{s}, V_{\varphi 0}, \chi\right)$. The error function was minimized using linear search optimization to determine $A^{S}, V_{\varphi 0}$ and $\chi$, where step 2 is utilized in each iteration to obtain the equilibrium values as indicated above.

\section{Results and discussion}

\subsection{Experimental measurements}

For the subsequent discussion, we use the nomenclature BHN to denote the formulations described in Table 2, where B represents BisGMA, $\mathrm{H}$ represents HEMA, and $\mathrm{N}$ is the percentage of BisGMA in the neat resin. For example, BH40 is the formulation corresponding to the yellow circle in Figure 1 and prepared by diluting the neat resin corresponding to the yellow square with water. The composition of a formulation lying on the phase boundary follows a well-defined curve, and hence is fully defined by the percentage of one of the components. Since we use formulations close to but not exactly on the phase boundary, the resin composition follows an approximate curve. We describe our results with respect to percentage of BisGMA in the resin. The following seven formulations - $\mathrm{BH} 85, \mathrm{BH} 70, \mathrm{BH} 55, \mathrm{BH} 40, \mathrm{BH} 25, \mathrm{BH} 20$ and $\mathrm{BH} 7.5$ were chosen to perform the absorption and swelling experiments. Figure 4 shows the measured data from water sorption, swelling and mechanical testing experiments.

Figure 4(a) shows the change of mass of the polymers with time in water obtained using Eq. 3 for both before and after pre-washing. The mass change in water increases after prewashing, which is likely due to the increase in free volume from washing away leachables. Figure 4(b) shows the volume change with time in water obtained using Eq. 4. From Figure 4(a) and (b) we observe that the rate of diffusion of water into the polymer as well as the rate of swelling decrease with increase in BisGMA along the phase boundary. Figure 4(c) and (d) show the final water sorption and volume change at equilibrium; these values also 
decrease with increase in BisGMA. This decrease in magnitude and the rate of water sorption is expected as discussed in our previous work [43], since with increasing BisGMA there is an increase in density of covalent crosslinks, non-covalent interactions and hydrophobicity.

Figure 4(e) shows the stoichiometric covalent crosslink density as well as the covalent crosslink density obtained from the rubbery modulus obtained using Eq. (5). Both these quantities increased with BisGMA content. However the crosslink density obtained from the rubbery modulus is several times larger than the stoichiometric crosslink density. The high cross-link density obtained from the rubbery modulus using Eq. (5), does not account for the shorter chain lengths in which the energetic contribution from chain stretching contributes significantly to the stiffness [86]. Models which account for these effects could potentially provide a better match between the crosslink densities calculated from the rubbery modulus and stoichiometry. This is however not the goal of this paper and we do not use either of these calculations for modeling, but have presented them for an understanding of the material behavior beyond glass transition.

Figure 4(f) shows the elastic modulus obtained under dry, wet and rubbery conditions with increasing BisGMA content. The modulus under wet conditions is smaller than that under dry conditions because of partial plasticization of non-covalent interactions upon imbibing water [42]. The non-covalent interactions are primarily hydrogen bonds, but may also include van der Waals interactions and physical entanglements. The non-covalent interactions exist between HEMA and BisGMA monomer units in the dry condition. In wet conditions, these interactions between HEMA monomer units get plasticized. However, the hydrogen bonding between BisGMA monomer units still exists due to the hydrophobic effect and provides the necessary stiffness under wet conditions. Beyond the glass transition temperature, all hydrogen bonding between the monomer units soften imparting freedom of movement to the polymer chains [87, 88]; hence we see that the rubbery modulus is orders of magnitude smaller than the moduli at room temperature at wet or dry conditions. These findings also underlines the significance of the micro-poro-mechanical model described in section 3.1, since the stiffness calculated from this model accounts for the contribution from not only the covalent cross-links between polymer segments but also the non-covalent hydrogen bonds, van der Waals interactions, entanglements, and other physical interactions, as well as the material porosity and swelling.

\subsection{Model calculations}

The numerical model calibration described in section 3.4 was used to obtain the meso-scale parameter $A^{s}$, the initial free volume $V_{\varphi 0}$, and the interaction parameter $\chi$. The calibration was performed for three different values of the parameter $\alpha^{s}: 0.2,0.5$ and 1, which represents the relative shear to normal stiffness in the theoretically dense polymer. Since $a^{s}$ $=0$ implies no shear interaction which is unrealistic for a crosslinked polymer, we have used $\alpha^{s}$ in the range of 0.2 to 1.0 such the Poisson's of theoretically dense polymer, $v^{s}$, ranges from 0 to 0.31 . In addition, we computed the Poisson's ratio, bulk modulus, poromechanics parameters and Lame's constants under undrained conditions at equilibrium. The variations of these properties with respect to percentage BisGMA were then examined to understand the structure-property relationships.

4.2.1 Meso-scale parameters-Figure 5(a) shows the variation of the initial free volume, $V_{\varphi 0}$, of the polymer with changing BisGMA concentration along the phase boundary. The free volume in a polymer depends on the available space between polymer chains, which is affected by a number of factors such as a) the nature of bonds between the polymer segments, $b$ ) the volume and distribution of water that was dissolved in the 
monomer mixture, c) evaporation of this dissolved water from the sample during curing, and d) the size distribution of the polymer segments. BisGMA increases the covalent cross-link density as well as the non-covalent interactions between the polymer segments, which are primarily hydrogen bonds, but may also include van der Waals interactions and physical entanglements. The presence of cross-linking may restrict the packing of chains and decrease the free volume [89]. Thus, with increasing crosslink density there is a smaller free volume, $V_{\varphi 0}$ to be saturated according to Eq. (17). So, the decrease in free volume from BH20 to BH85 may be attributed to this increased density of interactions. However, we observe that the free volume of BH7.5 is lower than that of the remaining formulations. This is possibly due to a decrease in free volume caused by a greater retention of the water molecules dissolved in the monomer, as bound water in the polymer, given the relatively high hydrophilicity of this formulation. We hypothesize that the influence of packing density on the free volume arising due to the dissimilar sizes of BisGMA and HEMA segments is outweighed by the other factors discussed here. We also observe that the model prediction of free volume decreases with decreasing $a_{s}$, however the trend in variation with BisGMA remains the same.

Further, in Figure 5(b) we show the equilibrium porosity, $\varphi$, or volumetric water content, $\gamma_{W}$. The volumetric water content depends upon both the hydrophobicity and the polymer network stiffness, and decreases with BisGMA content in a nonlinear fashion. As per Eq. (17), under equilibrium conditions when $\mu=0, \gamma_{W}$ has a nonlinear dependence on the fluid pressure as well as on $X$, which is representative of hydrophobicity. The fluid pressure in turn depends on the polymer network stiffness $C_{i j k l}$, as represented in Eq. (18). At low BisGMA content, the network stiffness is small and the hydrophobicity is low leading to high water absorption. The water absorption decreases with BisGMA content and asymptotes to around 7 percent.

The dimensionless interaction parameter $X$, originally introduced in [44] and [90] characterizes the energy of mixing of the solvent and polymer. The relationship between the polymer-water interaction parameter $X$ with the BisGMA content is shown in Figure 5(c). Overall, $X$ shows an increasing relationship with the percentage of BisGMA. The interaction parameter, $X$ depends on the following factors: a) interactions between water molecules and the polymer molecules by virtue of its definition, b) cross-link density of the polymer network, and c) the packing of the polymer chains and the overall structure. In particular, it is known that the $X$ for cross-linked polymer exceeds that of the corresponding un-crosslinked polymer [91]. An increasing trend of $X$ with the weight percent of the cross-linker was found in a study by [92] for swelling experiments on polymers formed from a hydrophobic/ hydrophilic monomer pair. Further, we find that the value of the interaction parameter $X$ for BH7.5 (2 mol\% BisGMA) obtained from our model ranges from 0.68 to 0.84 for $a_{s}$ going from 1.0 to 0.2 . The calculated values compare favorably with the measured value $(X=0.77)$ reported in [93] for polyHEMA with 2.5 mole \% crosslinker. We also note that the value of the interaction parameter $X$ for BH85 obtained from our model ranges from 1.96 to 2.19 for $a_{s}$ going from 0.2 to 1.0 . These values compare well with the interaction parameter $X$ for neat BisGMA $(X=2.29)$ computed using Hansen solubility parameter [94]. From Figure 5, we also see that $a_{S}=0.2$ or less leads to a decrease in the interaction parameter $X$ for BisGMA content between $20 \%$, to $60 \%$ which is contrary to expectation since BisGMA is more hydrophobic than HEMA. Therefore, $a_{s}=0.2$ is physically unrealistic for BisGMA content between $20 \%$, to $60 \%$. Overall an $a_{s}$ between 0.5 and 1 gives a more realistic estimate of $X$. It is also noteworthy that the monomer formulations lie along the phase boundary i.e. they contain HEMA, BisGMA as well as water. The amount and nature of water that remains in the polymer after the polymerization could also influence the variation of $X$. A greater amount of bound water present in the polymer would decrease the value of $X$ since it would facilitate mixing by decreasing the enthalpy of mixing of additional water. A 
similar decrease in $X$ has been experimentally observed as the water volume fraction in the polymer increases with swelling [47, 95-97].

From Figure 5(d) we observe that for all cases of $a_{s}$, the meso-scale parameter $A^{s}$ increases with BisGMA content. It is noteworthy that the parameter $A^{S}$ represents the average stiffness and number density of interactions between polymer segments. The increase in covalent crosslink density with BisGMA is clear from Figure 4(e). The increase of $A^{S}$ with BisGMA is not only contributed by the covalent crosslink density but also by the increase in noncovalent and other physical interactions as discussed earlier. As observed in Figure 4(f), the formulations BH7.5 and BH20 are nearly rubbery under wet conditions due to low crosslink density and resultant long chain lengths caused by the large HEMA content and corresponding plasticization by water. Hence, we observe a corresponding steep increase in $A^{s}$ at 20\% BisGMA content which is in agreement with the steep rise in stiffness with decreasing chain lengths reported rubber elasticity (see Chapter 1 of [86]). With further increase in BisGMA content, the contribution is increasingly energetic, and at the highest BisGMA content, the polymer is close to a crosslinked glass network, and $A^{s}$ asymptotes for constant $a^{s}$. Based on the results of the interaction parameter, we predict that $\alpha^{s}$. lies between 0.5 and 1 at BisGMA contents beyond $20 \%$, therefore the model predicts $A^{S}$ within a narrow band.

4.2.2 Macromechanical properties-Figure 6(a) shows the variation of undrained Poisson's ratio with percentage BisGMA. We observe from Figure 5(c) that the predicted interaction parameter $X$ are relatively insensitive to $\alpha^{s}$ for BisGMA content $<20 \%$ while $a^{s}$ between 0.5 and 1 provides realistic estimate for BisGMA content $>20 \%$. The predicted values of undrained Poisson's ratio form a narrow band for BisGMA content $<20 \%$ with values close to 0.5 for BH7.5. For BisGMA content $>20 \%$ the predicted values of undrained Poisson's ratio form a relatively broader band for $\alpha^{s}$ varying from 0.5 to 1.0. For BH85, the predicted undrained Poisson's ratio varies from 0.04 to 0.17 . We note that at high BisGMA concentrations, the water volume fraction is very low and the polymer is very close to the dry condition. For highly crosslinked glasses similar Poisson's ratios of $0.11-0.15$ have been determined under dry conditions [98]. We see that the model leads us to realistic predictions of Poisson's ratios using values of $\alpha^{s}$ that correspond to realistic predictions of $X$. This demonstrates the usefulness of the model in relating macro-scale mechanical properties with chemical composition. Further, we note that the decrease of Poisson's ratio with increase in crosslinking has also been experimentally observed [98].

We observe that at low BisGMA content, wherein the contribution of cross-linking is relatively small, the Poisson's ratio tends to 0.5 , which is same as that of water. From Figure 6(a), we see that for all values of $a_{s}$, the undrained Poisson's ratio $\nu^{\mu}$ for BH7.5 is in the range of 0.47 to 0.49 . It has been experimentally confirmed that the Poisson's ratio of hydrogels with low cross-link density is indeed close to 0.5. For example, [99] determined the Poisson's ratio of poly-HEMA hydrogel to be $0.42 \pm 0.03$ at $\mathrm{pH} 2.8$ and $0.45 \pm 0.12$ at $\mathrm{pH}$ 12 and [67] found Poisson's ratios of $0.48 \pm 0.012$ for relatively low cross-link density. The values of Poisson's ratio estimated from the model are particularly useful because the experimental determination of Poisson's ratio of these material is not straightforward and may involve specialized image analysis techniques [99]. Based upon the optimization scheme described in step 2 of section 3.4, we determined a Poisson's ratio of 0.489 at $7.5 \%$ BisGMA, which lies in the range of the experimental observations. Additional experimental measurements of shear modulus may be necessary for precise prediction of these properties.

The variation in bulk modulus with BisGMA content is also shown in Figures 6(b). Though the bulk modulus increases with the percentage of BisGMA, we observe that the bulk modulus for the saturated polymer is much lower than that of water or the dry polymer, 
which suggests that these materials cannot be modeled using simple mixture theories. Similar low values of bulk modulus in the range of $1 \mathrm{MPa}$ has been measured for polyHEMA gel under undrained conditions [67] and bulk moduli varying from $30 \mathrm{MPa}$ to $300 \mathrm{MPa}$ have been measured for articular cartilage [100, 101], which is a fiber reinforced hydrogel. Thus, the bulk moduli in general for hydrogels are much lower than that of water. The high value of Poisson's ratio and the low value of the bulk modulus at low BisGMA content are due to the low drained stiffness of the resultant highly porous polymer network. Particularly for the Poisson's ratio, the contributions of the poromechanical parameters dominate in the undrained conditions.

Figure 6(c) and (d) show the variation of the poromechanical parameters $B$ and $\Gamma$ with respect to the BisGMA content. The decreasing value of $B$ implies that the role of the fluid pressure in resisting the applied load decreases with the BisGMA percentage. The decreasing value of $\Gamma$ indicates decrease in overall compressibility of the saturated polymer with increase in BisGMA. The decrease in overall compressibility is attributed to increased covalent, non-covalent and physical interactions, as well as reduced water volume fraction leading to reduced plasticization with increase in BisGMA. Figure 6(e) and (f) give the variation of the Lame's constants for reference. The Lame's constants are an alternative method to express the material elastic constants. We observe that the model predicts that the shear modulus $\mu^{\mu}$ tends to zero at low BisGMA content. The macro-scale shear modulus arises from the resistance to shear between grains at the meso-scale. As the crosslink density decreases, the meso-scale shear resistance decreases, thus decreasing the macro-scale shear modulus. For a highly porous and water saturated hydrogel with low crosslink density, the model correctly predicts a low shear modulus that tends towards that of an ideal fluid. It is noteworthy that the model gives reasonable estimates for a number of properties, especially their trends with BisGMA content, using a relatively small number of experiments.

\section{Summary and Conclusions}

The dentin adhesives used in composite restorations polymerize in the presence of water and thus vary in their spatial composition, spanning about ten microns from the dentin end to the composite polymer end. Generally, these adhesives are a conglomerate of many angstromscale structural units, which interact through various covalent bonds forming linear as well as cross-links and non-covalent interactions such as van der Waals, hydrogen bonds, and physical interactions. Their molecular structure is typically deduced by polymer chemists based on the information from their chemistry of polymerization. Though the molecular structure of the monomers and the polymerization mechanism is qualitatively understood, the details of their meso-scale (sub-micron scale) structure, composed of polymer chains cross-linked at intervals and entanglements is not known. Due to the large number of atoms in a meso-scale, and the ill-defined knowledge of meso-structure and molecular interactions, there are severe limitations to atomistic-scale modeling (both molecular dynamics and $a b$ initio) of these material systems.

Therefore, in this paper we adopted the granular micromechanics approach, in which the material is treated as a granular system whose grain interactions represent the average behavior of the various molecular-scale interactions. In addition, we included chemo-poromechanical effects using a meso-scopic chemical potential and Biot's poromechanical methodology for incorporating the pore-fluid pressure. The developed model was applied to investigate the chemo-poro-mechanical properties of dentin adhesive polymer phases formed along the ternary adhesive-water phase boundary. These dentin adhesive polymer phases represent the composition of adhesive monomer applied to wet demineralized dentin. Water sorption, swelling, static and dynamic mechanical tests were performed. An optimization procedure was developed to determine the meso-scale model parameters using 
the experimentally measured sorption, swelling and elastic behaviors. These model parameters are identifiable with molecular scale interactions. The variation of these parameters along the phase boundary quantifies the effect of cross-link density and hydrophobicity on the chemo-poro-mechanical properties. It is noteworthy that the trends predicted by the model agreed with independent experimental measurements and with chemical compositions. The model was used to predict additional mechanical properties like Poisson's ratio and bulk modulus, which are difficult to measure experimentally. Further, we obtained insights into mechanisms at the molecular scale that are potentially responsible for the bulk material properties.

In the field of restorative dentistry, an intuitive approach based on experience is often used by polymer chemists to identify or design new co-monomer adhesive molecules to meet multiple target criteria such as water sorption and elastic modulus. We expect that the chemo-mechanical model introduced in this paper will enhance this approach by providing a stronger link between polymer chemistry and the macro-scale chemo-mechanical properties. Further, the model parameters have been determined along the adhesive-dentin phase boundary. These model parameters can be used for describing the hybrid layer mechanical properties for input into finite element models of dentin-adhesive interfaces. The model can also be extended to incorporate polymer rate-dependent behavior such that the fluid-flow and the intrinsic viscous effects can be distinguished resulting in a more comprehensive description of the mechanical behavior of these materials.

\section{Supplementary Material}

Refer to Web version on PubMed Central for supplementary material.

\section{Acknowledgments}

The authors gratefully acknowledge financial support for this project by National Institutes of Health/ National Institute of Dental and Craniofacial Research (R01DE014392, 3R01DE014392-08S1, and R01 DE022054).

\section{References}

1. Bao, Q-B.; Higham, PA. Google Patents. 1993. Hydrogel bead intervertebral disc nucleus.

2. Bao, Q-B.; Higham, PA. Google Patents. 1996. Hydrogel intervertebral disc nucleus with diminished lateral bulging.

3. Dai W, Barbari T. Gel-impregnated pore membranes with mesh-size asymmetry for biohybrid artificial organs. Biomaterials. 2000; 21:1363-71. [PubMed: 10850930]

4. Giraud MN, Ayuni E, Cook S, Siepe M, Carrel TP, Tevaearai HT. Hydrogel-based Engineered Skeletal Muscle Grafts Normalize Heart Function Early After Myocardial Infarction. Artificial organs. 2008; 32:692-700. [PubMed: 18684206]

5. Hoffman AS. Hydrogels for biomedical applications. Advanced drug delivery reviews. 2012

6. Inoue T, Chen G, Nakamae K, Hoffman AS. A hydrophobically-modified bioadhesive polyelectrolyte hydrogel for drug delivery. Journal of controlled release. 1997; 49:167-76.

7. Middleton JC, Tipton AJ. Synthetic biodegradable polymers as orthopedic devices. Biomaterials. 2000; 21:2335-46. [PubMed: 11055281]

8. Roulet, JF.; Degrange, M. Adhesion: The silent revolution in dentistry. Quintessence Pub Co; 2000.

9. Roy S, Prabhakar B. Bioadhesive polymeric platforms for transmucosal drug delivery systems-a review. Tropical Journal of Pharmaceutical Research. 2010:9.

10. Wang X, Sui S. Pulsatile Culture of a Poly (DL-Lactic-Co-Glycolic Acid) Sandwiched Cell/ Hydrogel Construct Fabricated Using a Step-by-Step Mold/Extraction Method. Artificial Organs. 2011; 35:645-55. [PubMed: 21671960] 
11. Yanagi K, Ookawa K, Mizuno S, Ohshima N. Performance of a new hybrid artificial liver support system using hepatocytes entrapped within a hydrogel. ASAIO transactions/American Society for Artificial Internal Organs. 1988; 35:570-2. [PubMed: 2597536]

12. Bharadwaj R, Mehrabi A, Hamilton C, Trujillo C, Murga M, Fan R, et al. Structure-property relationships in cross-linked polyester-clay nanocomposites. Polymer. 2002; 43:3699-705.

13. Shonaike, GO.; Advani, SG. Advanced polymeric materials: structure property relationships. CRC Press; 2003.

14. Van Krevelen, DW.; Te Nijenhuis, K. Properties of polymers: their correlation with chemical structure; their numerical estimation and prediction from additive group contributions: Access Online via Elsevier. 2009.

15. Nakabayashi N, Ashizawa M, Nakamura M. Identification of a resin-dentin hybrid layer in vital human dentin created in vivo: durable bonding to vital dentin. Quintessence international (Berlin, Germany: 1985). 1992; 23:135.

16. Nakabayashi N, Nakamura M, Yasuda N. Hybrid Layer as a Dentin-Bonding Mechanism. Journal of Esthetic and Restorative Dentistry. 1991; 3:133-8.

17. Zou, Y. Adhesive resin conversion and composition in the hybrid layer of the resin-dentin bond. Iowa City: University of Iowa; 2007.

18. Zou Y, Armstrong SR, Jessop JLP. Quantitative analysis of adhesive resin in the hybrid layer using Raman spectroscopy. Journal of Biomedical Materials Research Part A. 2010; 94:288-97. [PubMed: 20186729]

19. Spencer P, Ye Q, Park J, Topp EM, Misra A, Marangos O, et al. Adhesive/dentin interface: the weak link in the composite restoration. Annals of biomedical engineering. 2010; 38:1989-2003. [PubMed: 20195761]

20. Peumans M, Kanumilli P, De Munck J, Van Landuyt K, Lambrechts P, Van Meerbeek B. Clinical effectiveness of contemporary adhesives: a systematic review of current clinical trials. Dental Materials. 2005; 21:864-81. [PubMed: 16009415]

21. Purk JH, Dusevich V, Glaros A, Eick JD. Adhesive analysis of voids in class II composite resin restorations at the axial and gingival cavity walls restored under in vivo versus in vitro conditions. Dental Materials. 2007; 23:871-7. [PubMed: 16950506]

22. Shin TP, Yao X, Huenergardt R, Walker MP, Wang Y. Morphological and chemical characterization of bonding hydrophobic adhesive to dentin using ethanol wet bonding technique. Dental Materials. 2009; 25:1050-7. [PubMed: 19371945]

23. Swift, EJ., Jr; Vargas, MA. Dentin/Enamel Adhesives: Their Current Status.

24. Talungchit, S. Enhancing resin-dentin bond effectiveness and durability: the role of ethanol-wet bonding technique, MMP-inhibition (chlorhexidine), and photoinitiator systems. 2012.

25. Carvalho RM, Ciucchi B, Sano H, Yoshiyama M, Pashley DH. Resin diffusion through demineralized dentin matrix. Revista de Odontologia da Universidade de São Paulo. 1999; 13:417-24.

26. Eick J, Gwinnett A, Pashley D, Robinson S. Current concepts on adhesion to dentin. Critical Reviews in Oral Biology \& Medicine. 1997; 8:306-35. [PubMed: 9260046]

27. Pashley D, Carvalho R. Dentine permeability and dentine adhesion. Journal of Dentistry. 1997; 25:355-72. [PubMed: 9241954]

28. Ye Q, Park J, Laurence JS, Parthasarathy R, Misra A, Spencer P. Ternary phase diagram of model dentin adhesive exposed to over-wet environments. Journal of dental research. 2011; 90:1434-8. [PubMed: 21960682]

29. Ye Q, Park J, Parthasarathy R, Pamatmat F, Misra A, Laurence JS, et al. Quantitative analysis of aqueous phase composition of model dentin adhesives experiencing phase separation. Journal of Biomedical Materials Research Part B: Applied Biomaterials. 2012

30. Hosaka K, Nakajima M, Takahashi M, Itoh S, Ikeda M, Tagami J, et al. Relationship between mechanical properties of one-step self-etch adhesives and water sorption. Dental Materials. 2010; 26:360-7. [PubMed: 20053432]

31. Hosaka K, Tagami J, Nishitani Y, Yoshiyama M, Carrilho M, Tay FR, et al. Effect of wet vs. dry testing on the mechanical properties of hydrophilic self-etching primer polymers. European journal of oral sciences. 2007; 115:239-45. [PubMed: 17587300] 
32. Ito S, Hashimoto M, Wadgaonkar B, Svizero N, Carvalho RM, Yiu C, et al. Effects of resin hydrophilicity on water sorption and changes in modulus of elasticity. Biomaterials. 2005; 26:6449-59. [PubMed: 15949841]

33. Malacarne J, Carvalho RM, de Goes MF, Svizero N, Pashley DH, Tay FR, et al. Water sorption/ solubility of dental adhesive resins. Dental Materials. 2006; 22:973-80. [PubMed: 16405987]

34. Sano H, Ciucchi B, Matthews W, Pashley D. Tensile properties of mineralized and demineralized human and bovine dentin. Journal of Dental Research. 1994; 73:1205-11. [PubMed: 8046110]

35. Sano H, Yoshikawa T, Pereira PNR, Kanemura N, Morigamui M, Tagami J, et al. Long-term durability of dentin bonds made with a self-etching primer, in vivo. Journal of dental research. 1999; 78:906-11. [PubMed: 10326735]

36. Yiu CK, Pashley EL, Hiraishi N, King NM, Goracci C, Ferrari M, et al. Solvent and water retention in dental adhesive blends after evaporation. Biomaterials. 2005; 26:6863-72. [PubMed: 15964621]

37. Yiu CKY, King NM, Carrilho MRO, Sauro S, Rueggeberg FA, Prati C, et al. Effect of resin hydrophilicity and temperature on water sorption of dental adhesive resins. Biomaterials. 2006; 27:1695-703. [PubMed: 16226310]

38. Misra A, Spencer P, Marangos O, Wang Y, Katz JL. Micromechanical analysis of dentin/adhesive interface by the finite element method. Journal of Biomedical Materials Research Part B: Applied Biomaterials. 2004; 70:56-65.

39. Misra A, Spencer P, Marangos O, Wang Y, Katz JL. Parametric study of the effect of phase anisotropy on the micromechanical behaviour of dentin-adhesive interfaces. Journal of The Royal Society Interface. 2005; 2:145.

40. Singh V, Misra A, Marangos O, Park J, Ye Q, Kieweg SL, et al. Fatigue life prediction of dentinadhesive interface using micromechanical stress analysis. Dental Materials. 2011; 27:E187-E95. [PubMed: 21700326]

41. Singh V, Misra A, Marangos O, Park J, Ye Q, Kieweg SL, et al. Viscoelastic and fatigue properties of model methacrylate based dentin adhesives. Journal of Biomedical Materials Research Part B: Applied Biomaterials. 2010; 95:283-90.

42. Singh V, Misra A, Parthasarathy R, Ye Q, Park J, Spencer P. Mechanical properties of methacrylate-based model dentin adhesives: Effect of loading rate and moisture exposure. Journal of biomedical materials research Part B, Applied biomaterials. 201310.1002/jbm.b.32963

43. Parthasarathy R, Misra A, Park J, Ye Q, Spencer P. Diffusion coefficients of water and leachables in methacrylate-based crosslinked polymers using absorption experiments. Journal of Materials Science: Materials in Medicine. 2012:1-16.

44. Flory PJ. Thermodynamics of high polymer solutions. The Journal of chemical physics. 1942; 10:51.

45. Flory, PJ. Principles of polymer chemistry. Cornell University Press; 1953.

46. Flory PJ, Rehner J Jr. Statistical Mechanics of Cross-Linked Polymer Networks II. Swelling The Journal of chemical physics. 1943; 11:521.

47. Horkay F, McKenna G. Polymer networks and gels. Physical Properties of Polymers Handbook. 2007:497-523.

48. Haward, RN.; Young, RJ. The physics of glassy polymers. Springer; 1997.

49. Park JG, Ye Q, Topp EM, Lee CH, Kostoryz EL, Misra A, et al. Dynamic mechanical analysis and esterase degradation of dentin adhesives containing a branched methacrylate. Journal of Biomedical Materials Research Part B: Applied Biomaterials. 2009; 91:61-70.

50. Krongauz VV. Diffusion in polymers dependence on crosslink density. Journal of thermal analysis and calorimetry. 2010; 102:435-45.

51. Alves NM, Ribelles JLG, Tejedor JAG, Mano JF. Viscoelastic behavior of poly(methyl methacrylate) networks with different cross-linking degrees. Macromolecules. 2004; 37:3735-44.

52. De Gennes, PG. Scaling concepts in polymer physics. Cornell university press; 1979.

53. Benoit H, Decker D, Duplessix R, Picot C, Rempp P, Cotton J, et al. Characterization of polystyrene networks by small-angle neutron scattering. Journal of Polymer Science: Polymer Physics Edition. 1976; 14:2119-28. 
54. Schulmann N, Meyer H, Wittmer J, Johner A, Baschnagel J. Interchain monomer contact probability in two-dimensional polymer solutions. Macromolecules. 2012; 45:1646-51.

55. Hazony D, Hazony Y, Katz JL, Welsch G. Average acoustic pulse dispersion length in condensed matter channels. Philosophical Magazine. 2006; 86:3043-60.

56. Chang CS, Chao SJ, Chang Y. Estimates of elastic moduli for granular material with anisotropic random packing structure. International journal of solids and structures. 1995; 32:1989-2008.

57. Misra A, Chang CS. Effective Elastic-Moduli of Heterogeneous Granular Solids. International Journal of Solids and Structures. 1993; 30:2547-66.

58. Misra A, Singh V. Micromechanical model for viscoelastic materials undergoing damage. Continuum Mechanics and Thermodynamics. 2013; 25:343-58.

59. Federico S, Herzog W. Towards an analytical model of soft biological tissues. Journal of Biomechanics. 2008; 41:3309-13. [PubMed: 18922533]

60. Ateshian GA, Rajan V, Chahine NO, Canal CE, Hung CT. Modeling the Matrix of Articular Cartilage Using a Continuous Fiber Angular Distribution Predicts Many Observed Phenomena. J Biomech Eng-T Asme. 2009:131.

61. Misra A, Parthasarathy R, Singh V, Spencer P. Poromechanics Parameters of Fluid-Saturated Chemically Active Fibrous Media Derived from a Micromechanical Approach. Journal of Nanomechanics and Micromechanics. 2013130531210358001.

62. Doi, M. Introduction to polymer physics. Oxford University Press on Demand; Oxford, UK: 1996.

63. de Gennes P-G. Granular matter: a tentative view. Reviews of modern physics. 1999; 71:S374.

64. Doi M. Gel Dynamics. Journal of the Physical Society of Japan. 2009:78.

65. Vandoolaeghe W, Terentjev E. Constrained Rouse model of rubber viscoelasticity. The Journal of chemical physics. 2005; 123:034902.

66. Jongschaap RJJ, Doeksen D. The Average Stress Tensor in Systems with Interacting Particles. Rheol Acta. 1983; 22:4-11.

67. Boudou T, Ohayon J, Picart C, Tracqui P. An extended relationship for the characterization of Young's modulus and Poisson's ratio of tunable polyacrylamide gels. Biorheology. 2006; 43:7218. [PubMed: 17148855]

68. Greaves GN, Greer AL, Lakes RS, Rouxel T. Poisson's ratio and modern materials. Nature Materials. 2011; 10:823-37.

69. Marra SP, Ramesh KT, Douglas AS. Mechanical characterization of active poly(vinyl alcohol)poly(acrylic acid) gel. Materials Science \& Engineering C-Biomimetic and Supramolecular Systems. 2001; 14:25-34.

70. Urayama K, Takigawa T, Masuda T. Poisson Ratio of Poly(Vinyl Alcohol) Gels. Macromolecules. 1993; 26:3092-6.

71. Klein CA, Cardinale GF. Youngs Modulus and Poissons Ratio of Cvd Diamond. Diamond and Related Materials. 1993; 2:918-23.

72. Wen Q, Basu A, Janmey PA, Yodh AG. Non-affine deformations in polymer hydrogels. Soft matter. 2012; 8:8039-49. [PubMed: 23002395]

73. Misra A, Marangos O, Parthasarathy R, Spencer P. Micro-scale Analysis of Compositional and Mechanical Properties of Dentin Using Homotopic Measurements. Biomedical Imaging and Computational Modeling in Biomechanics. 2013:131-41.

74. Eshelby JD. The determination of the elastic field of an ellipsoidal inclusion, and related problems Proceedings of the Royal Society of London Series A Mathematical and Physical Sciences. 1957; 241:376-96.

75. Dormieux L, Molinari A, Kondo D. Micromechanical approach to the behavior of poroelastic materials. Journal of the Mechanics and Physics of Solids. 2002; 50:2203-31.

76. Quiligotti S, Maugin GA, dell'Isola F. An Eshelbian approach to the nonlinear mechanics of constrained solid-fluid mixtures. Acta Mechanica. 2003; 160:45-60.

77. Mura, T. Micromechanics of defects in solids. Springer; 1987.

78. Misra A, Parthasarathy R, Singh V, Spencer P. Micro-poromechanics model of fluid-saturated chemically active fibrous media. Zeitschrift für Angewandte Mathematik und Mechanik. 2013:120.10.1002/zamm.201300071 
79. dell'Isola F, Madeo A, Seppecher P. Boundary conditions at fluid-permeable interfaces in porous media: A variational approach. International Journal of Solids and Structures. 2009; 46:3150-64.

80. Madeo A, Dell'Isola F, Ianiro N, Sciarra G. A variational deduction of second gradient poroelasticity II: An application to the consolidation problem. Journal of Mechanics of Materials and Structures. 2008; 3:607-25.

81. Sciarra G, Dell'Isola F, Ianiro N, Madeo A. A variational deduction of second gradient poroelasticity part I: General theory. Journal of Mechanics of Materials and Structures. 2008; 3:507-26.

82. Yasuda H, Peterlin A, Colton CK, Smith KA, Merrill EW. Permeability of solutes through hydrated polymer membranes. Part III. Theoretical background for the selectivity of dialysis membranes. Die Makromolekulare Chemie. 2003; 126:177-86.

83. Amsden B. Solute diffusion within hydrogels. Mechanisms and models Macromolecules. 1998; 31:8382-95.

84. Tauer K, Ali AI, Sedlak M. On the preparation of stable poly (2-hydroxyethyl methacrylate) nanoparticles. Colloid and Polymer Science. 2005; 283:351-8.

85. Xu X, Goponenko AV, Asher SA. Polymerized polyHEMA photonic crystals: $\mathrm{pH}$ and ethanol sensor materials. Journal of the American Chemical Society. 2008; 130:3113-9. [PubMed: 18271586]

86. Mark, JE.; Erman, B. Science and technology of rubber. Academic Press; 2011.

87. Ashby, MF.; Jones, DRH. Engineering Materials 2: An Introduction to Microstructures and Processing. Butterworth-Heinemann; 2012.

88. Safranski DL, Gall K. Effect of chemical structure and crosslinking density on the thermomechanical properties and toughness of (meth) acrylate shape memory polymer networks. Polymer. 2008; 49:4446-55.

89. Khonakdar HA, Morshedian J, Wagenknecht U, Jafari SH. An investigation of chemical crosslinking effect on properties of high-density polyethylene. Polymer. 2003; 44:4301-9.

90. Huggins ML. Solutions of long chain compounds. The Journal of chemical physics. 1941; 9:440.

91. Freed KF, Pesci AI. Computation of the crosslink dependence of the effective Flory interaction parameter. chi. for polymer networks. Macromolecules. 1989; 22:4048-50.

92. Wang J, Wu W. Swelling behaviors, tensile properties and thermodynamic studies of water sorption of 2-hydroxyethyl methacrylate/epoxy methacrylate copolymeric hydrogels. European polymer journal. 2005; 41:1143-51.

93. Lee JW, Kim EH, Jhonf MS. The Swelling and Mechanical Properties of Hydrogels of Tactic Poly (2-Hydroxyethyl Methacrylate). Bulletin of Korean Chemical Society. 1983; 4:162-9.

94. Hansen, CM. Hansen solubility parameters: a user's handbook. New York: CRC Press; 2000.

95. Fomenko A, Pospíšil H, Sedlakova Z, Pleštil J, Ilavský M. Phase transition in swollen gelsPart 32. Temperature transition in charged poly (N-isopropylmethacrylamide) hydrogels in water and aqueous $\mathrm{NaCl}$ solutions. Physical Chemistry Chemical Physics. 2002; 4:4360-7.

96. Nagy M. A comparative and systematic thermodynamic study of aqueous solutions and hydrogels of homo-and copolymers of poly (vinyl alcohol). Physical Chemistry Chemical Physics. 2000; 2:2613-22.

97. Shafee EE, Naguib H. Water sorption in cross-linked poly (vinyl alcohol) networks. Polymer. 2003; 44:1647-53.

98. Rouxel T. Elastic Properties and Short-to Medium-Range Order in Glasses. Journal of the American Ceramic Society. 2007; 90:3019-39.

99. Johnson B, Bauer JM, Niedermaier DJ, Crone WC, Beebe DJ. Experimental techniques for mechanical characterization of hydrogels at the microscale. Experimental mechanics. 2004; 44:218.

100. Milentijevic D, Torzilli PA. Influence of stress rate on water loss, matrix deformation and chondrocyte viability in impacted articular cartilage. Journal of biomechanics. 2005; 38:493-502. [PubMed: 15652547] 
101. Oloyede A, Flachsmann R, Broom ND. The dramatic influence of loading velocity on the compressive response of articular cartilage. Connective tissue research. 1992; 27:211-24. [PubMed: 1576822] 

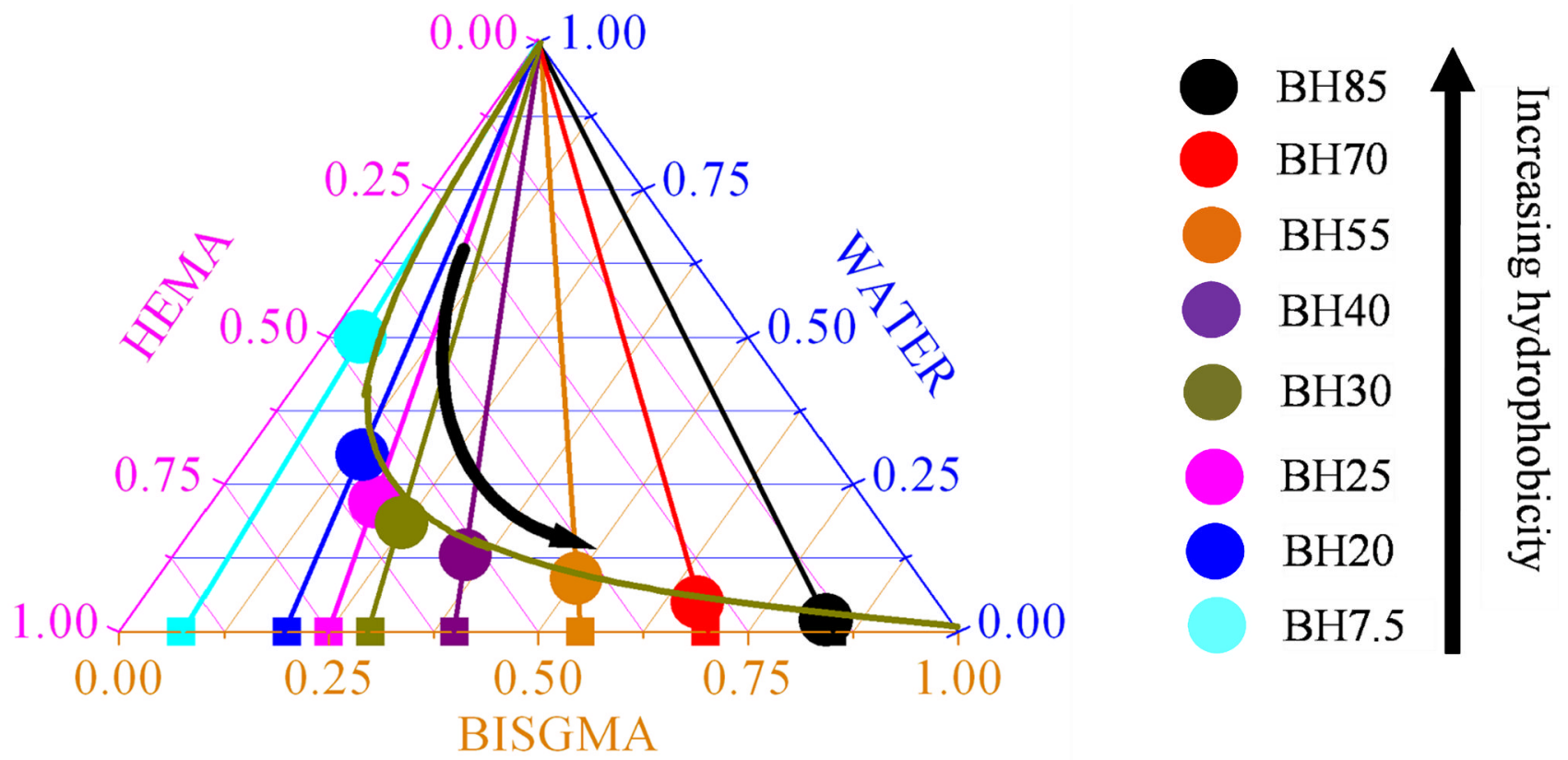

Figure 1.

(Color online) Representative adhesive monomer formulations identified on the phase boundary of the water-adhesive ternary phase diagram. The circles represent the formulations prepared close to the phase boundary. The squares represent the corresponding neat formulations. 

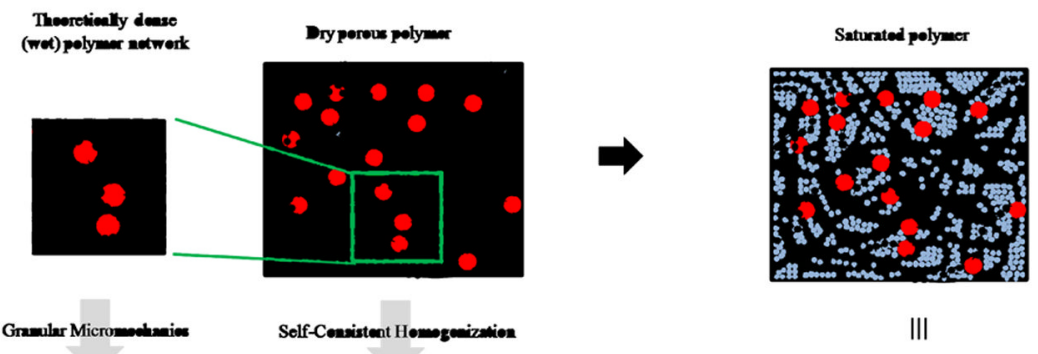

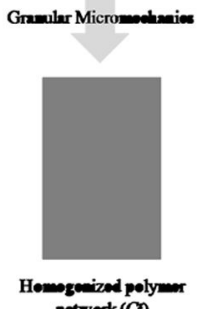

(a)

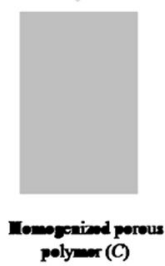

(b)

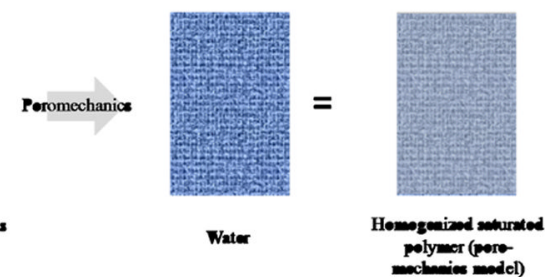

(c)

Figure 2.

(Color online) Schematic depicting steps for micro-poro-mechanical modeling. 


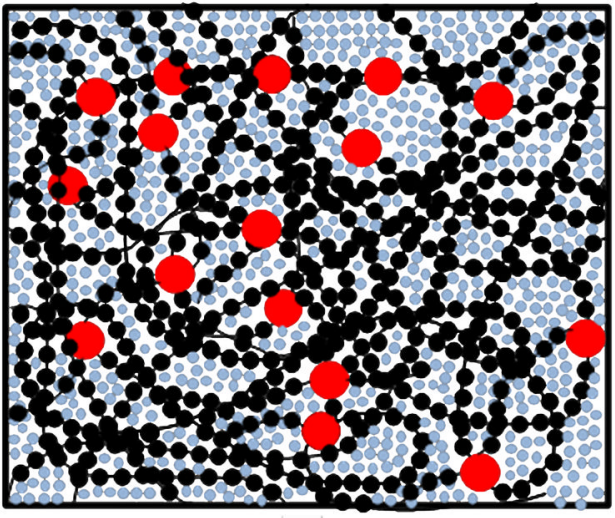

(a)

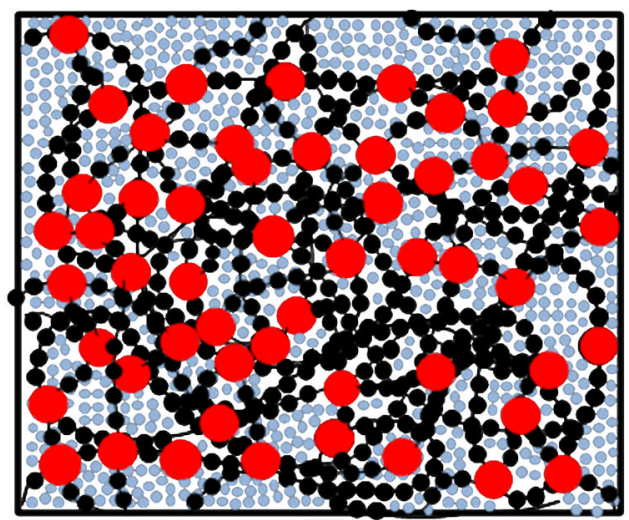

(b)

Figure 3.

(Color online) Schematic of the saturated polymer for (a) sparsely cross-linked network and (b) highly cross-linked network. The red molecule belongs to the cross-links and the black molecules to linear polymer chains. The large molecule in the polymer is a BisGMA unit and the smaller one is a HEMA unit. The blue molecules represent water in the interstitial spaces between the polymer chains. 

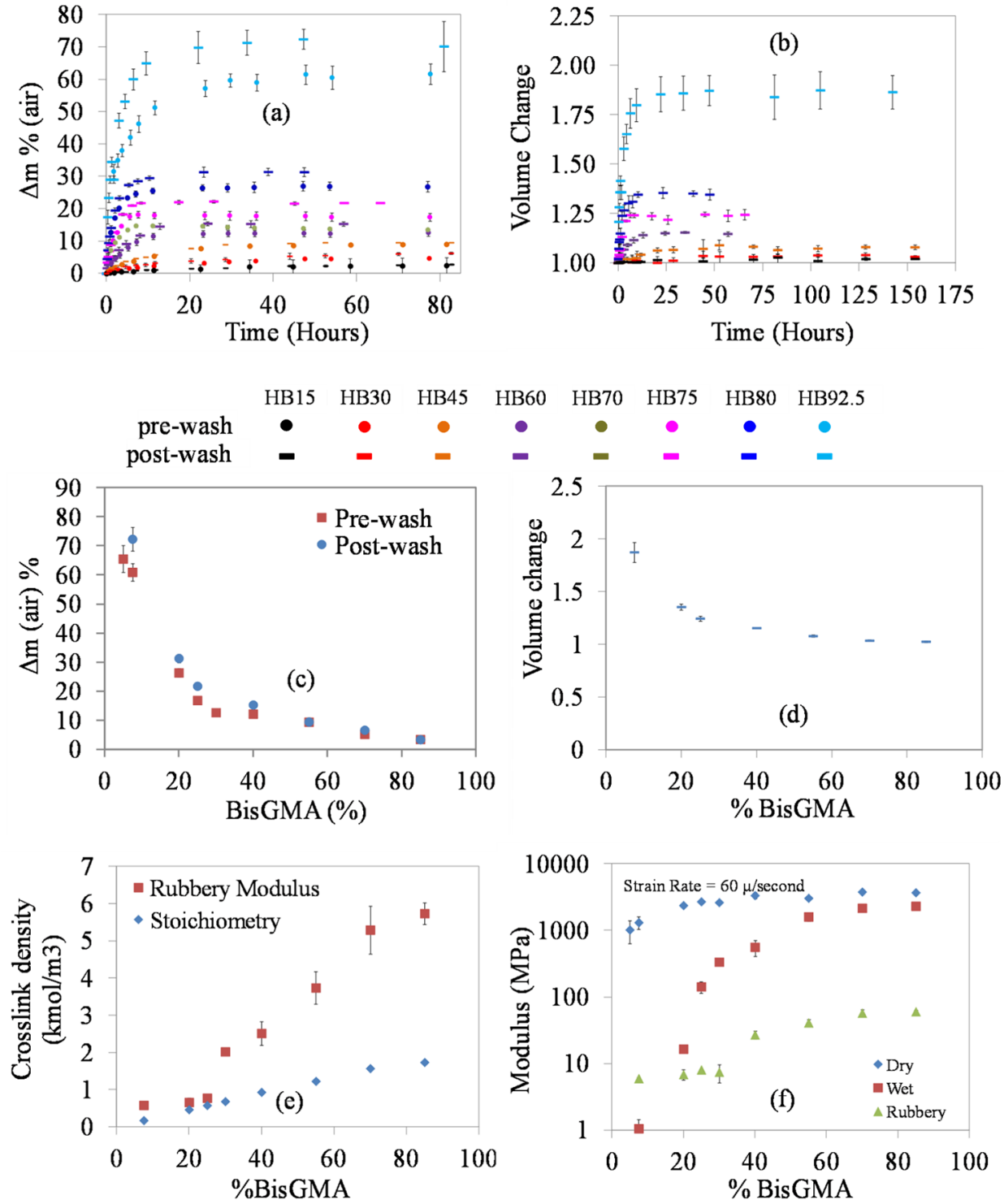

Figure 4.

(Color online) Experimental measurements on polymer formulations: (a) pre-wash and postwash change of mass in air with time, (b) post-wash change of volume with time, (c) prewash and post-wash change of mass in air at equilibrium, (d) post-wash volume change at equilibrium, (e) crosslink density obtained from rubbery modulus and stoichiometry, and (f) elastic modulus under dry, wet and rubbery conditions. 

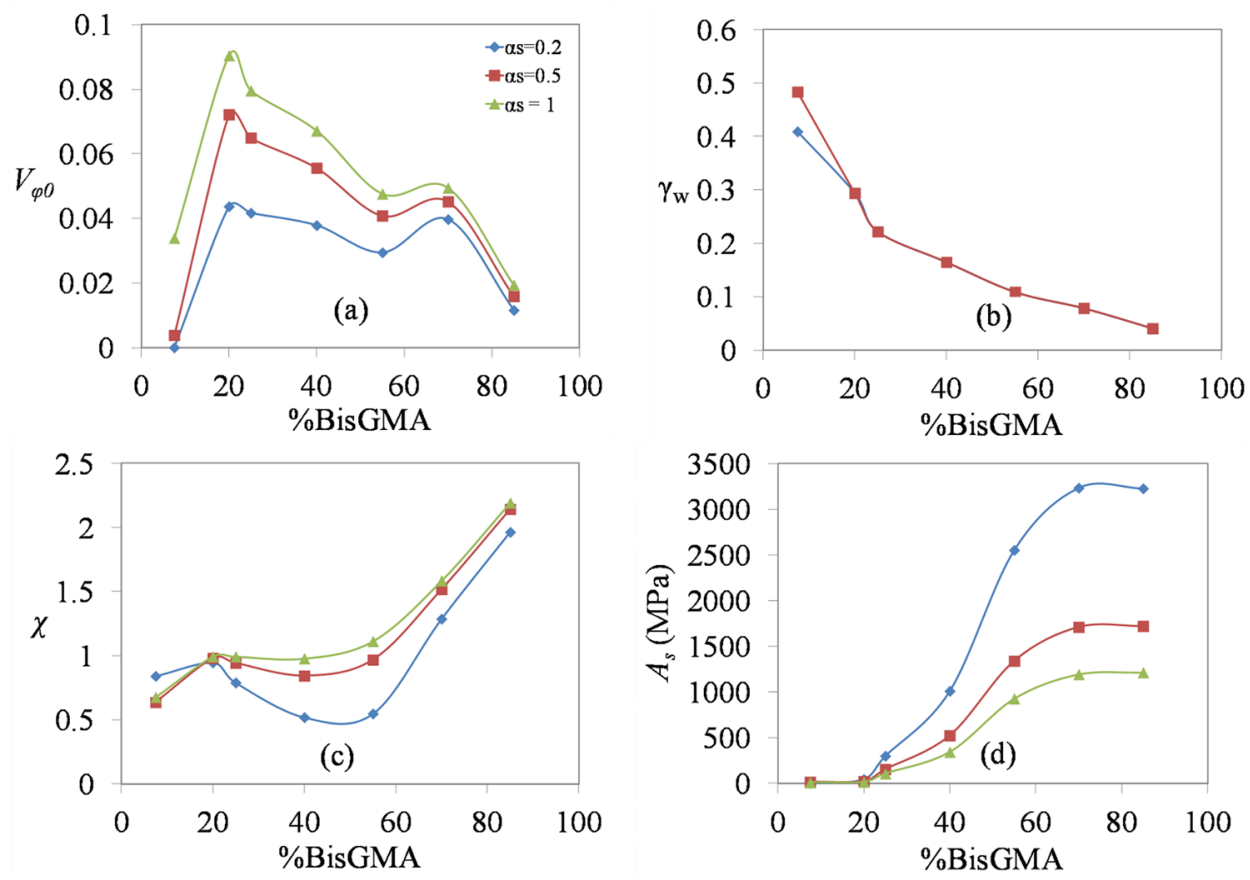

Figure 5.

(Color online) Variation of model parameters with percentage of BisGMA: a) free volume, b) volumetric water fraction, c) interaction parameter, and d) meso-scale parameter representing average inter-granular stiffness and density. 

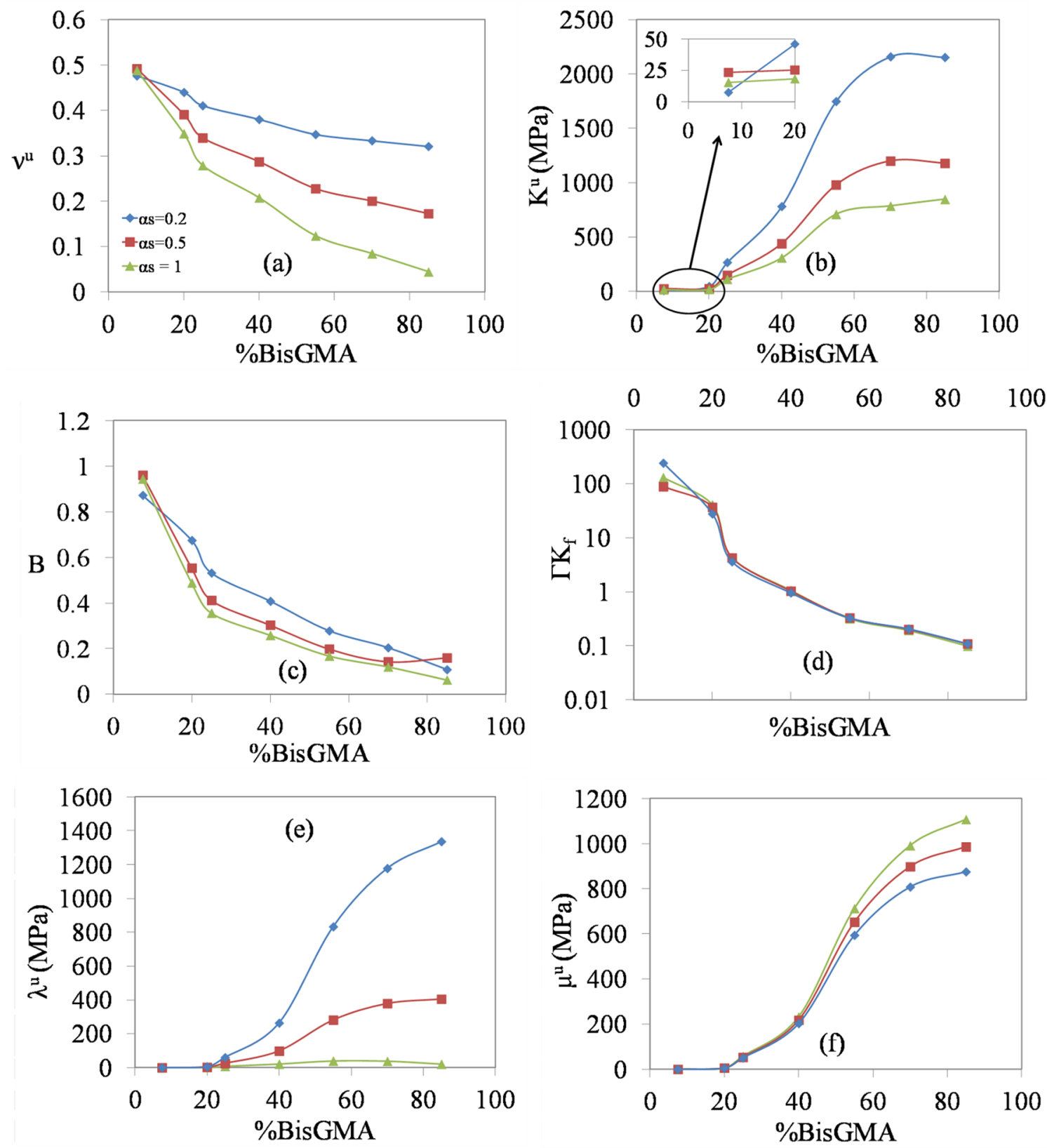

Figure 6.

(Color online) Correlation between derived mechanical properties and percentage of BisGMA: (a) Poisson's ratio under undrained conditions, (b) bulk modulus under undrained conditions, (c) poromechanics constant B, (d) material compressibility relative to water, (e) Lame's first parameter under undrained conditions, and (e) Lame's second parameter (shear modulus) under undrained conditions. 
Table 1

List of co-monomers.

\begin{tabular}{|c|c|}
\hline Co-monomer chemical formula/name & Co-monomer Structure \\
\hline BisGMA & \\
\hline HEMA & \\
\hline
\end{tabular}

Supplier: Sigma Chemical Co., St. Louis, MO, USA. 
Table 2

Percentage composition of the monomer formulations on the phase boundary

\begin{tabular}{|c|c|c|c|c|}
\hline & BisGMA (\%) & HEMA (\%) & Water (\%) & Miscibility (\%) \\
\hline BH85 & 0.833 & 0.147 & 0.02 & -- $^{*}$ \\
\hline BH70 & 66.51 & 28.51 & 4.98 & 6.69 \\
\hline BH55 & 50.05 & 40.95 & 9 & 10.65 \\
\hline BH40 & 34.8 & 52.2 & 13 & 15.25 \\
\hline BH30 & 24.45 & 57.05 & 18.5 & 21.14 \\
\hline BH25 & 19.5 & 58.5 & 22 & 24.82 \\
\hline BH20 & 14 & 56 & 30 & 32.48 \\
\hline BH7.5 & 3.75 & 46.25 & 50 & 53.76 \\
\hline
\end{tabular}

Too viscous to measure miscibility 
Table 3

Relation between meso-scale parameters and macromechanical material properties

\begin{tabular}{|c|c|c|}
\hline Macro-mechanical property & & $(\boldsymbol{A}, \boldsymbol{\alpha})$ \\
\hline Elastic modulus & $E$ & $A\left(\frac{\alpha}{2+3 \alpha}\right)$ \\
\hline Poisson's Ratio & $\mathrm{v}$ & $\frac{1-\alpha}{2+3 \alpha}$ \\
\hline Bulk modulus & $\mathrm{K}$ & $\frac{2 A}{3}$ \\
\hline Shear modulus & $\mu$ & $5 A\left(\frac{\alpha}{3+2 \alpha}\right)$ \\
\hline Lame's first parameter & $\lambda$ & $A\left(\frac{1+\alpha}{2+3 \alpha}\right)$ \\
\hline
\end{tabular}

Note: Superscripts $s$ and $u$ used in the text refer to the theoretically dense wet polymer network and the saturated swollen polymer under undrained conditions. 